\title{
Variabilités et Tendances des Paramètres Hydroclimatiques dans le Bassin Versant de la Rivière Banco au Sud de la Côte d'Ivoire
}

Kone Souleymane, Doctorant en Géosciences et Environnement

Université Nangui Abrogoua, Côte d'Ivoire

Bamba Siaka Barthelemy, Chargé de recherche

Centre de Recherches Océanologiques, Côte d'Ivoire

Ouattara Ismaila, Assistant

Université Nangui Abrogoua, Côte d'Ivoire

Université de Man, Côte d'Ivoire

Diallo Seydou, Doctorant en Géosciences et Environnement

Université Nangui Abrogoua, Côte d'Ivoire

Kamagaté Bamory, Maître de Conférence

Université Nangui Abrogoua, Côte d'Ivoire

Université de Man, Côte d'Ivoire

Doi:10.19044/esj.2019.v15n27p282 URL:http://dx.doi.org/10.19044/esj.2019.v15n27p282

\section{Résumé}

La présente étude menée sur le bassin versant de la rivière Banco à Abidjan $\left(5^{\circ} 20^{\prime}\right.$ ' et $5^{\circ} 26^{\prime}$ de latitude Nord et entre $4^{\circ} 1^{\prime}$ et $4^{\circ} 5^{\prime}$ de longitude Ouest) dans le sud de la Côte d'Ivoire vise à mettre en exergue l'existence d'une variabilité climatique, à partir des fluctuations et des tendances dans les données hydroclimatiques (pluies, températures et débits). Pour atteindre cet objectif, diverses données (pluviométriques, thermométriques et hydrométriques) et méthodes (indice de Nicholson, tests de Cusum, de tstudent, de Mann-Kendall et de l'estimateur de la pente de Sen) ont été utilisées. A la lumière des résultats acquis, une variabilité climatique caractérisée par une alternance d'années humides, normales et sèches a été mise en évidence dans la zone d'étude. Les indices et méthodes statistiques montrent la continuité de la récession pluviométrique établie depuis 1970 avant une reprise pluviométrique à partir de l'année 2000. En ce qui concerne les températures, on assiste à une légère baisse de la température moyenne annuelle provoquée par la régression des températures minimales. La température moyenne dans le bassin versant a baissé de $1,4^{\circ} \mathrm{C}$ au cours des 34 dernières années. Les manifestations de cette variabilité climatique affectent peu le débit de la rivière. Une augmentation importante de l'écoulement 
superficielle apparaît dans le bassin avec un taux de changement de 71,25\%, indiquant ainsi une bonne disponibilité en eau.

Mots-clés: Côte d'Ivoire, Bassin versant de la rivière Banco, Variabilité climatique, Mann-Kendall, Pente de Sen

\section{Variability and Trends of Hydroclimatic Parameters in the Banco River Watershed in Southern Ivory Coast}

Kone Souleymane, Doctorant en Geosciences et Environnement

Université Nangui Abrogoua, Côte d'Ivoire

Bamba Siaka Barthelemy, Charge de recherche

Centre de Recherches Océanologiques, Côte d'Ivoire

Ouattara Ismaila, Assistant

Université Nangui Abrogoua, Côte d'Ivoire

Université de Man, Côte d'Ivoire

Diallo Seydou, Doctorant en Geosciences et Environnement

Université Nangui Abrogoua, Côte d'Ivoire

Kamagate Bamory, Maitre de Conference

Université Nangui Abrogoua, Côte d'Ivoire

Université de Man, Côte d'Ivoire

\section{Abstract}

This study conducted in the basin of Banco river in Abidjan ( $5^{\circ} 20^{\prime}$ and $5^{\circ} 26^{\prime}$ at latitude north and between $4^{\circ} 1^{\prime}$ and $4^{\circ} 5^{\prime}$ at longitude west) in the south of Cote d'Ivoire aims at underlining the existence of climate variability on the basis of fluctuations and trends in hydro climatic data ( rainfalls, temperatures and discharges). To reach this goal, diverse data (pluviometric, thermometric and hydrometrical) and methods (Nicholson rating/index, Mann Kendall test; Cusum test; t-student test and Sen incline/slope estimator) are used. In light of the results, a climatic variability characterized by the alternation of humid, normal and dry years have been revealed in the area under study. The statistical indexes/ratings and methods show the continuity of the rainfall recession established since 1970 before a resumption of the pluviometry from the year 2000 onwards. Concerning the temperatures, we notice a slight fall of the annual average temperature triggered by the 
regression of minimal temperatures. The average temperature in the hole basin has decreased of $1.4^{\circ} \mathrm{C}$ in the last 34 years. The manifestations of this climate variability hardly impact on the flow of the river. An important increase in the superficial outflow appears in the basin with a rate of change of $71.25 \%$, indicating therefore sufficient water availability.

Keywords: Côte d'Ivoire, Banco river basin, Climate variability, Mannkendall, Sen's slope

\section{Introduction}

Les impacts du changement climatique se matérialisent majoritairement à travers l'eau (inondations et sécheresses) (Zerouali et al., 2015).Le changement climatique affecte la configuration pluviométrique à long terme, la disponibilité de l'eau et le risque d'apparition de graves sécheresses et inondations. Mais, également la croissance démographique et le développement des activités anthropiques sur les bassins versants constituent une véritable menace pour leur fonctionnement. D'où la nécessité de comprendre la dynamique des variations actuelles qui affectent les ressources en eau afin de les protéger pour un développement durable. Tel est le cas du bassin versant de la rivière Banco.

Située dans l'aire de recharge de la nappe d'Abidjan, la forêt du Banco sert de protection naturelle à une partie de la nappe d'eau souterraine qui alimente la ville d'Abidjan et couvre approximativement le bassin d'une petite rivière, le Banco, qui se jette dans la lagune Ebrié à la sortie de la forêt. Aussi, intervient-elle dans la conservation des ressources naturelles (flore, faune, sol, eau...) et sert d'espace pour l'animation pédagogique, la recherche, l'écotourisme et l'éducation environnementale. Elle favorise une bonne pluviométrie et a un rôle de purification de l'air. Il convient de mentionner que la nappe du continental terminal affleure dans cette zone, la rendant potentiellement vulnérable à la pollution (Kouassi et al., 2010). Cependant, les études hydroclimatiques des petits bassins versants du littoral ivoirien sont rares, à fortiori celles consacrées au bassin versant du Banco qui datent des années 1980 (Louroux, 1978; Jourda, 1987). Il faut noter de ces anciens travaux que la méthodologie d'étude de la variabilité climatique n'est plus adéquate. Les études sur le changement climatique sont principalement axées sur l'importance des changements probables des paramètres hydroclimatiques. La variabilité de ces paramètres peut être étudiée à l'aide d'une approche statistique basée sur l'analyse de données climatiques à long terme (Patle et al., 2014). Ainsi, l'indice de Nicholson et les tests de ruptures (Cusum et t-student) sont adéquats pour déterminer les variations dans les séries de données, et apprécier le degré de sécheresse. A l'effet de détecter des tendances dans les séries hydroclimatiques, le test de Mann-kendall couplé à 
la méthode de la pente de Sen a été utilisé. Le test de Mann-Kendall est une méthode robuste largement utilisée pour analyser les tendances dans les séries temporelles (Mann, 1945; Kendall, 1955). Plusieurs chercheurs ont utilisé cette approche pour différents paramètres hydrométéorologiques (Fossou et al.,2015; Faye, 2016; Yao et al., 2018). Dans ce bassin, caractéristique de l'écosystème forestier tropical humide, la récession pluviométrique des dernières décennies fait peser une menace sur les ressources en eau. C'est dans ce contexte que cette étude, à échelle spatiale réduite, permettra de mieux apprécier les fluctuations climatiques.

\section{Zone d'étude et données}

\subsection{Localisation de la zone d'étude}

Le bassin versant de la rivière Banco est localisé au Nord d'Abidjan, précisément entre les latitudes $5^{\circ} 20^{\prime} \mathrm{N}$ et $5^{\circ} 26^{\prime} \mathrm{N}$ et longitudes $4^{\circ} 1^{\prime} \mathrm{W}$ et $4^{\circ} 5^{\prime} \mathrm{W}$. Il couvre une superficie totale de $53 \mathrm{~km}^{2}$ entre les zones urbaines des communes de Yopougon au Sud-ouest, d'Attécoubé au Sud, d'Adjamé au Sud-est et celle d'Abobo au Nord-est et au Nord (Figure 1). Le climat est de type équatorial de transition (climat Attiéen). La pluviométrie annuelle est de $2100 \mathrm{~mm}$ et la température moyenne annuelle est de $26,2^{\circ} \mathrm{C}$ (Durand et Chantraine,1982). La rivière du Banco avec une profondeur moyenne d'un mètre $(1 \mathrm{~m})$ et une longueur de $9 \mathrm{~km}$ (Loroux, 1978) est caractérisée par un régime hydrologique de type équatorial de transition. Ce type de régime est marqué par le dédoublement de la crue annuelle. Le relief est constitué de plateaux à faible pente et de vallées à pente forte à fond plat. Les plateaux d'altitudes comprises entre $50 \mathrm{~m}$ et $100 \mathrm{~m}$ sont entrecoupés de vallées aux pentes très marquées. Le contexte géologique de la zone d'étude est celui du continental terminal ivoirien correspondant à des formations d'âge MioPliocène se caractérisant par une stratification lenticulaire de sables grossiers, d'argiles bariolées, de grès ferrugineux et de minerais de fer. Le bassin est constitué en majeure partie d'un vestige de forêt tropicale dense humide (WCMC, 1991) qui s'étend sur une superficie de 3474 hectares (Sako et al., 2013).

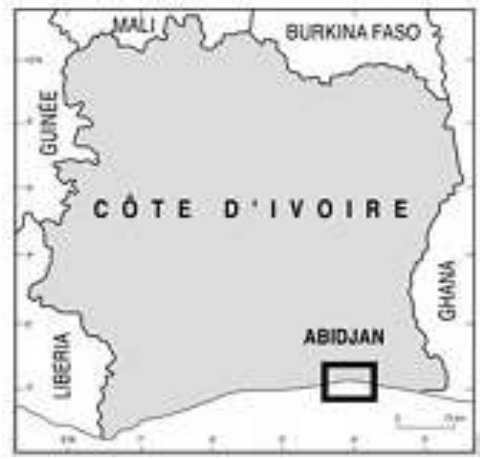




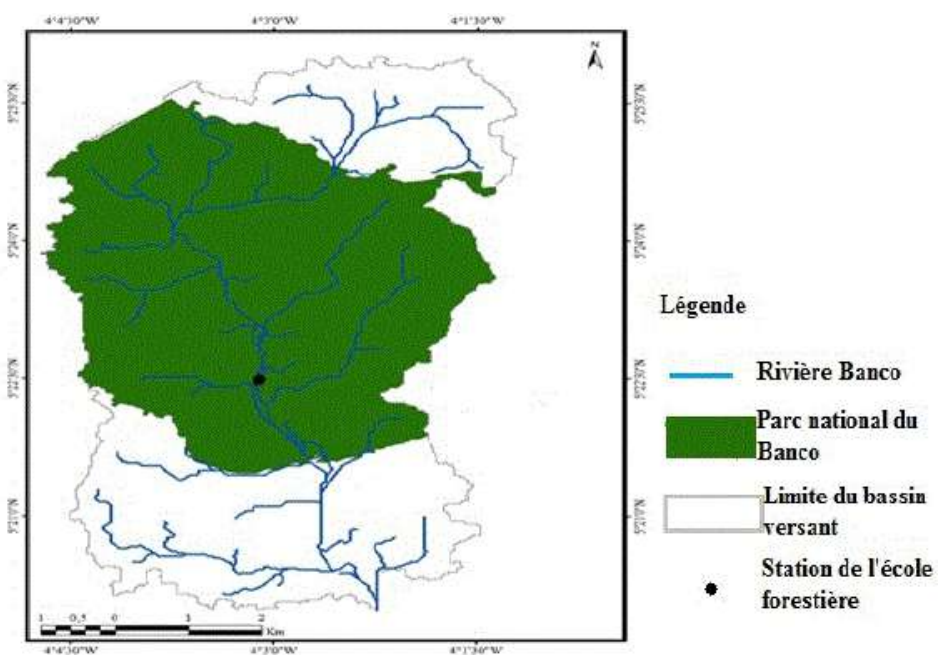

Figure1 : Situation géographique du bassin versant de la rivière Banco

\subsection{Données de l'étude}

L'étude repose principalement sur deux types de données: climatiques et hydrologiques. Les données climatiques, représentées par les températures et les précipitations sur une période de 34 ans (1980-2013), proviennent de la station agro-climatologique d'Adiopodoumé du CNRA (Centre National de Recherches Agronomiques). Les données de températures et de pluies journalières ont été compilées de manière à calculer les valeurs mensuelles, saisonnières et annuelles. La raison du choix de cette station est dueau fait qu'elle a été régulièrement suivie jusqu'à nos jours et présente une série chronologique d'une longueur supérieure à 30 années d'observations ininterrompues. Cette station semble assez représentative de la pluviométrie enregistrée dans la région d'étude, et offre une meilleure garantie au niveau de la qualité des observations enregistrées.

Les données hydrologiques sont des débits au pas de temps journalier sur la même période (1980-2013) enregistrées à la station nommée «école forestière du banco» pris comme exutoire dans cette étude. Ces données de débits proviennent de la Direction de l'Hydraulique Humaine (DHH). Le bassin de la rivière banco dispose d'une seule station hydrométrique dont la durée d'observation s'étend de 1979 à 2013.

\section{Méthodes}

\subsection{Méthode d'identification des fluctuations}

L'étude de la fluctuation se fera principalement à travers le calcul d'indice hydroclimatique (Equation 1) et les tests statistiques de rupture et de tendance à l'aide du logiciel Trend (Version 1.0.2). 


\subsubsection{Indice de Nicholson}

Le calcul des indices hydroclimatiques à travers la formule de Nicholson (Nicholson, 1980; Servat et al., 1997) a pour objectif de mettre en évidence la variabilité interannuelle et de dégager les grandes tendances des variables étudiées. Ces indices ont permis de découper les séries de la station selon les années excédentaires ou déficitaires et d'en connaitre les grandes tendances.

$$
I=\frac{x_{i}-\bar{x}}{\sigma}
$$

Avec $x_{i}$ : hauteur annuelle du paramètre étudié pour la station au cours d'une année i;

$\bar{x}$ : moyenne interannuelle à la station;

$\sigma$ : écart type de la série.

\subsubsection{Tests de détection des ruptures dans les séries chronologiques}

\subsubsection{Test de Cusum}

Le test de Cusum permet de vérifier la stationnarité des séries hydroclimatiques. Cette méthode consiste à diviser en deux échantillons chaque série étudiée avant d'examiner si les moyennes dans deux échantillons de la série de données sont différentes. C'est un test non paramétrique suivant les données d'une série chronologique $(x 1, x 2, x 3, \ldots . ., x n)$. Le test statistique est défini comme suit:

$$
\begin{aligned}
V \mathrm{~K}=\sum_{i=1}^{\mathrm{K}} \operatorname{sgn}\left(x_{i}-x_{\text {médian }}\right) \\
\mathrm{K}=1,2,3, \ldots \ldots, n
\end{aligned}
$$

$\operatorname{sgn}(\mathrm{x})=\left\{\begin{array}{c}1 \text { pour } x>0 \\ 0 \text { pour } x=0 \\ -1 \text { pour } x<0\end{array}\right.$

$x_{\text {médian }}$ est la valeur médiane de l'ensemble de la série de données. La distribution de $V \kappa$ suit la loi de kolmogorov-smirnov à deux échantillons (Ks= $(2 / n)$ maximum $|V k|)$ avec la valeur seuil du maximum $|V k|$ donnée par:
$\alpha=\left\{\begin{array}{l}0,10 \\ 0,05 \\ 0,01\end{array}\right.$
$1,22 \sqrt{n}$

Une valeur négative de $V k$ indique que la dernière partie de la chronique à une moyenne plus élevée que la partie précédente et vice versa.

\subsubsection{Test de $\mathbf{t}$ - student}

Ce test paramétrique repose sur des comparaisons de moyennes. Cette méthode examine si les moyennes dans deux périodes distinctes sont différentes. Le test suppose que les données sont normalement distribuées. La formule statistique de $\mathrm{t}$-Student est la suivante: 


$$
t=\frac{(\bar{X}-\bar{Y})}{\sqrt[s]{\frac{1}{n}+\frac{1}{m}}}
$$

Où $\bar{X}$ et $\bar{Y}$ sont les moyennes de la prémière et de la seconde période; $\mathrm{m}$ et $\mathrm{n}$ sont les nombres d'observations respectifs de ces périodes et $\mathrm{S}$ est l'écart type de l'échantillon.

\subsubsection{Tests de tendance dans les séries statistiques}

L'évaluation des tendances a été réalisée à l'aide du logiciel Trend (V1.0.2) qui utilise le test Mann-Kendall (pour la détection des tendances et de leur signification statistique). Le test de Mann-Kendall est une méthode non paramétrique (Mann, 1945; Kendall, 1955) basée sur la détection d'une tendance dans des données chronologiques. Il a pour objectif d'évaluer statistiquement s'il existe une tendance monotone à la hausse ou à la baisse de la variable considérée dans le temps. Une tendance monotone à la hausse ou à la baisse signifie que la variable augmente ou diminue de manière constante au fil du temps, mais cette tendance peut être linéaire ou non. La statistique de Mann-Kendall notée $S$ s'écrit:

$$
S=\sum_{i=1}^{n-1} \sum_{j=i+1}^{n} \operatorname{sgn}(X j-X i)
$$

Où $X i, X j$ sont les valeurs de données séquentielles, $\mathrm{n}$ est la longueur de l'ensemble des données et

$$
\operatorname{sgn}(D)=\left\{\begin{array}{c}
1 \text { pour } D>0 \\
0 \text { pour } D=0 \\
-1 \text { pour } D<0
\end{array}\right.
$$

La valeur de $\mathrm{S}$ indique le sens de la tendance. Une valeur négative (positive) indique une tendance à la baisse (à la hausse). Lorsque $n \geq 8$, la statistique de test $S$ est approximativement normalement distribuée avec une moyenne et une variance comme suit:

$$
\begin{aligned}
& E(S)=0 \\
& \operatorname{Var}(S)=\frac{n(n-1)(2 n+5)-\sum_{i=1}^{m} T i(T i-1)(2 T i+5)}{18}
\end{aligned}
$$

La statistique de test normalisé $\mathrm{Z}$ est calculée comme suit:

$$
Z M K=\left\{\begin{array}{l}
\frac{s-1}{\sqrt{\operatorname{Var}(S)}}, \text { pour } S>0 \\
0 \text { pour } S=0 \\
\frac{S+1}{\sqrt{\operatorname{Var}(S)}}, \text { pour } S<0
\end{array}\right.
$$

Où $n$ est le nombre de groupes liés et $T i$ la taille du groupe de départ. En outre, ZMK suit la distribution normale, un ZMK positif indique une tendance à la hausse et un ZMK négatif, une tendance à la baisse pour la période. Au niveau de signification $\alpha, Z M K \geq Z \alpha / 2$, la tendance des données est alors significative. Le test de Mann Kendall vérifie l'hypothèse nulle d'absence de tendance par rapport à l'hypothèse alternative d'existence d'une tendance des données 
(Kumar et Jain, 2010). L’équation (8) est valable lorsque le nombre d'observations $\mathrm{n} \geq 10$.

\subsection{Estimation de l'amplitude des tendances}

Le test de tendance de Mann Kendall indique simplement la présence d'une tendance, mais ne montre aucune indication sur l'ampleur de celle-ci. Alors, l'estimateur de la pente de Sen (Sen, 1968) est utilisé pour déterminer l'ampleur de la tendance. Selon Gocic et Trajkovic (2013), la pente de Sen est une formule utilisée pour mesurer l'ampleur de la tendance en estimant la pente de la tendance linéaire supposée. Lorsque la série chronologique de données montre une tendance linéaire, une pente vraie peut être estimée en utilisant une procédure simple non paramétrique donnée par Sen. Cette méthode consiste à calculer les pentes de toutes les données de la série. Ensuite à estimer la pente de Sen par la pente médiane.

$$
T i=\frac{X j-X k}{J-K} \operatorname{sii}=1,2,3, \ldots, N
$$

Où $\mathrm{Xj}$ et $\mathrm{Xk}$ sont les valeurs de données pour $\mathrm{j}$ et $\mathrm{k}$ fois d'une période où $\mathrm{j}>\mathrm{k}$. la pente est estimée pour chaque observation. La médiane est calculée à partir des observations $\mathrm{N}$ de la pente pour l'estimateur de la pente de Sen:

$$
\begin{aligned}
& Q i=T \frac{N+1}{2} \text { si } N \text { est impair } \\
& Q i=\left(T^{N} \frac{N}{2}+T^{N+1} \frac{1}{2}\right) \text { si } N \text { est pair }
\end{aligned}
$$

Lorsque les observations de la pente $\mathrm{N}$ apparaissent en tant qu'impair, l'estimateur de Sen est calculé sous la forme Qmed $=(N+1) / 2$ et, pour les moments pairs, l'estimation de la pente sous la forme Qmed $=[(\mathrm{N} / 2)+((\mathrm{N}$ +2) / 2)] / 2. Le test bilatéral est effectué à $100(1-\alpha) \%$ de l'intervalle de confiance pour obtenir la pente vraie du test non-paramétrique de la série. La pente positive ou négative Qi est obtenue en montant (en augmentation) ou à la baisse (décroissante).

\section{Résultats et discussion}

\subsection{Résultats}

\subsubsection{Fluctuation des paramètres hydroclimatiques}

\subsubsection{Variabilité de la pluviométrie}

Les tests de t-Student (paramétrique) et de Cusum (non paramétrique) appliqués à la série des pluies (tableau 1) confirment la présence d'une rupture de stationnarité en 1999 au seuil de 0,1. Ils permettent d'identifier deux (2) grandes périodes: une période sèche (1980-1999), avec une moyenne pluviométrique de $3528,54 \mathrm{~mm}$ qui est légèrement inférieure à la moyenne annuelle totale $(3566,44 \mathrm{~mm})$; une période humide (2000-2013), avec une moyenne pluviométrique de $3620,57 \mathrm{~mm}$ qui est supérieure à la moyenne annuelle totale. Cette date est confirmée par la variation interannuelle du SPI 
(figure 2) qui indique l'année 1999 comme la limite entre une période antérieure sèche et une période postérieure humide.

Par ailleurs, nous pouvons relever 3 années sèches $(-1.5<\mathrm{SPI}<-1)$; 1980, 1998 et 2012 et 5 années humides (1982, 1987, 1996, 2000 et 2010). Les années 1982 et 2010 sont extrêmement humides (SPI>2). Ainsi, cette représentation de la fluctuation par l'indice normalisé des précipitations (SPI) reflète bien la variabilité pluviométrique de la station d'Adiopodoumé.

Tableau 1: Tests statistiques de rupture appliqués aux séries chronologiques.

\begin{tabular}{|c|c|c|c|c|}
\hline \multirow[t]{3}{*}{ Paramètre } & \multicolumn{4}{|c|}{ Test de rupture } \\
\hline & \multicolumn{2}{|c|}{ Cusum } & \multicolumn{2}{|c|}{ t-Student } \\
\hline & $\mathrm{V}_{\mathrm{k}}$ & Stat $\alpha=0,1$ & $\mathrm{t}$ & Stat $_{\alpha=0,1}$ \\
\hline \multirow[t]{2}{*}{ Pluie } & 6 & \multirow[t]{2}{*}{7} & \multirow[t]{2}{*}{$-0,803$} & \multirow[t]{2}{*}{1,651} \\
\hline & (1999) & & & \\
\hline \multirow{3}{*}{ Température } & $\mathrm{V}_{\mathrm{k}}$ & Stat $_{\alpha=0,1}$ & $\mathrm{t}$ & Stat $\alpha=0,01$ \\
\hline & 5 & \multirow[t]{2}{*}{7} & \multirow[t]{2}{*}{$-0,006$} & \multirow[t]{2}{*}{$2,736^{*}$} \\
\hline & $(1998 ; 2004)$ & & & \\
\hline \multirow{3}{*}{ Débit } & $\mathrm{V}_{\mathrm{k}}$ & Stat $\alpha=0,01$ & $\mathrm{t}$ & Stat $\alpha=0,01$ \\
\hline & 14 & \multirow[t]{2}{*}{$10^{*}$} & \multirow[t]{2}{*}{$-0,0003$} & \multirow[t]{2}{*}{$2,7^{*}$} \\
\hline & (1996) & & & \\
\hline
\end{tabular}

*rupture significative au seuil de 0,01.

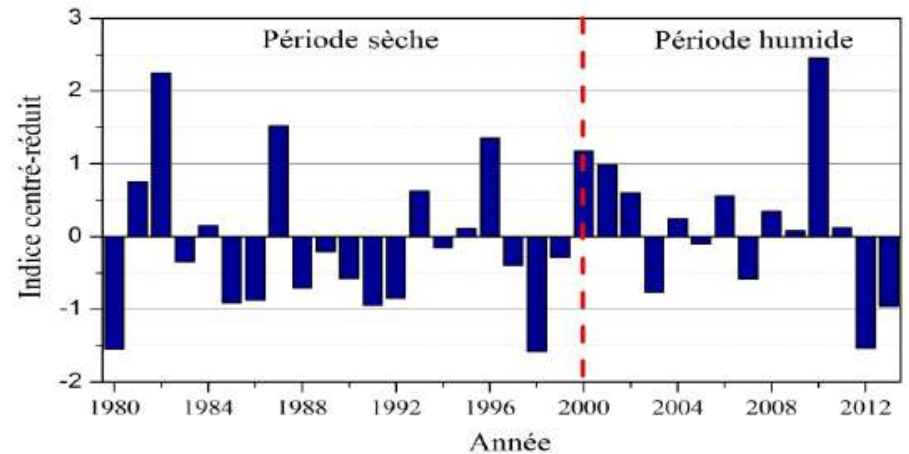

Figure 2: Variation de l'indice pluviométrique à la station d'Adiopodoumé de 1980 à 2013

La pluviométrie annuelle moyenne du bassin pendant la période de l'étude est de 3566,44 $\mathrm{mm}$ avec un écart-type de 290,14 $\mathrm{mm}$ et un faible coefficient de variation (CV) de $8,14 \%$ (Figure 3). Les précipitations minimales et maximales enregistrées sont de 3109,8 mm en 1998 (année la plus sèche) et $4277,4 \mathrm{~mm}$ en 2010 (année la plus humide). Une légère remontée de la moyenne des précipitations annuelles calculées à partir de 2000 est observable sur la chronique.Cependant, les années 1980, 1998 et 2012 sont les plus déficitaires. 


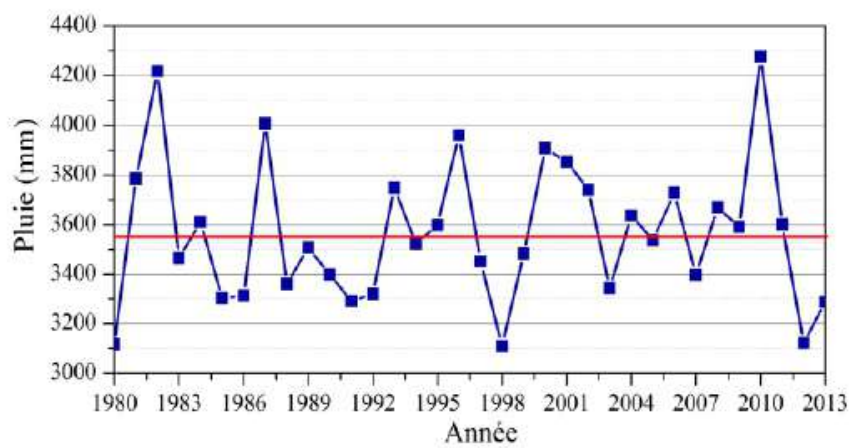

Figure 3:Evolution de la pluie moyenne interannuelle à la station d'Adiopodoumé (1980 à 2013)

A l'échelle mensuelle, les précipitations moyennes les plus élevées sont enregistrées en juin $(387,52 \mathrm{~mm})$ puis en mai $(231,71 \mathrm{~mm})$. En revanche, les précipitations les plus faibles sontobservées en janvier et en février avec respectivement $24,66 \mathrm{~mm}$ et $40,56 \mathrm{~mm}$. La distribution saisonnière a en réalité deux maximas: le premier en juin (grande saison des pluies), comme décrit cidessus, et le second en octobre (petite saison des pluies) avec une moyenne de 180,23 mm de pluie (Figure 4).

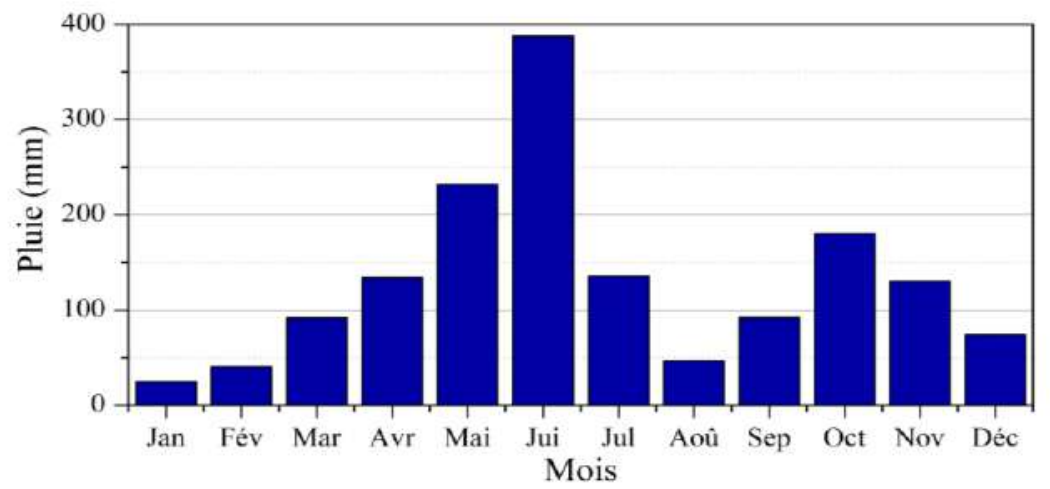

Figure 4: Evolution de la pluie moyenne mensuelle à la station d'Adiopodoumé (19802013)

\subsubsection{Variabilité de la température}

L'année 1998 est indiquée, comme année de rupture à la fois par les tests de Cusum et de t-Student (tableau 1). La moyenne $\left(23,4^{\circ} \mathrm{C}\right)$ avant 1998 est légèrement supérieure à la moyenne après $1998\left(23,03^{\circ} \mathrm{C}\right) . \quad \mathrm{La}$ représentation graphique de l'indice centré-réduit des températures interannuelles (figure 5) indique bien l'année 1998 comme date de rupture. Par ailleurs, cette figure indique l'année 2004 comme une rupture de stationnarité dans la série de température. Cette année est confirmée par le test de t-Student au seuil de 0,01 (tableau 1). 
Les anomalies interannuelles de la température enregistrées sur la figure 5 indiquent l'existence de deux périodes chaudes (1980-1998 et 20052013) séparées d'une période froide (1999-2004). De plus, l'année 2003 est exceptionnellement «froide » alors que les années 1987 et 2010 sont exceptionnellement «chaudes ». Il faut aussi retenir l'année 1998 comme une année chaude et les années 2002, 2003, 2004 et 2013 comme des années froides. Ce fait montre la possibilité de variation thermique interannuelle.

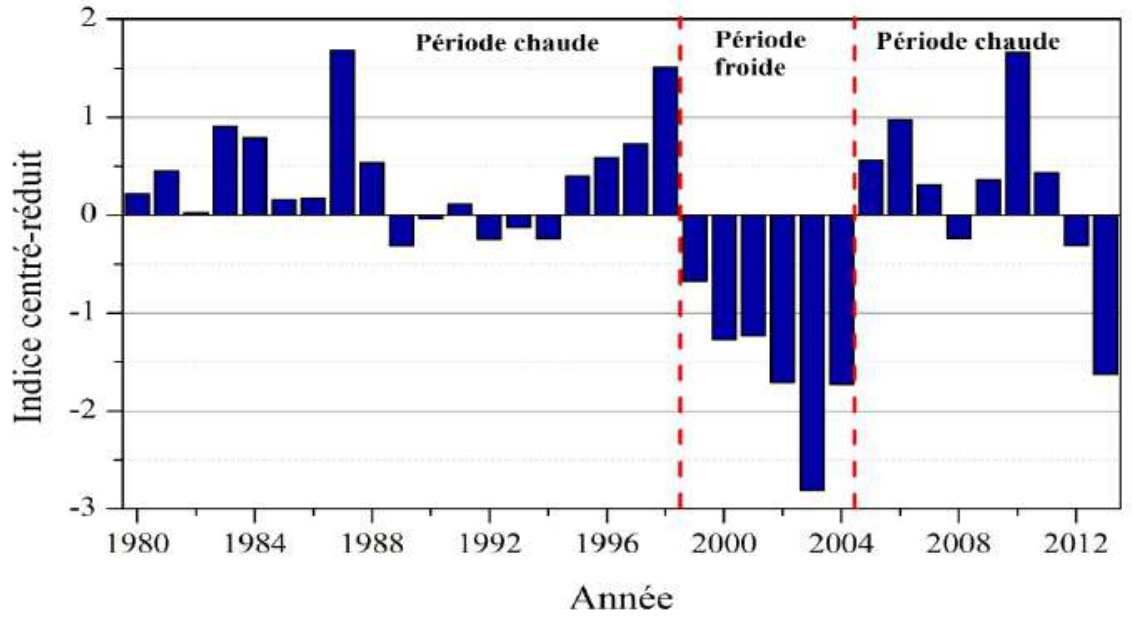

Figure 5:Anomalies de températures moyennes à Adiopodoumé sur la période 1980-2013

Les variations interannuelles de la température de l'air montrent que la température connaît une légère baisse sur la période 1980-2013. La température est restée supérieure à la moyenne $\left(23,2{ }^{\circ} \mathrm{C}\right)$ sur les périodes chaudes (1980-1998 et 2005-2013) et inférieure à cette valeur entre 1999 et 2004. On assiste à une alternance de période chaude et de période froide au niveau des variables (Tmoy, Tmax et Tmin) de cette station (Figure 6). Cependant, le refroidissement semble avoir été plus important pour les températures minimales que pour les températures maximales. L'écart-type est de $0,5^{\circ} \mathrm{C}$ par rapport à la moyenne globale, ce qui permet de dire que la variabilité interannuelle est faible par rapport au réchauffement sur la période. La température maximale dans le bassin versant varie de $23,1^{\circ} \mathrm{C}$ (avril 2003) à $26^{\circ} \mathrm{C}$ (mars 1998), la température maximale annuelle moyenne étant de $24,52^{\circ} \mathrm{C}$ avec un écart type de $0,64^{\circ} \mathrm{C}$. La température minimale dans le bassin versant varie de $18,78^{\circ} \mathrm{C}$ (mai 2003) à $23,32^{\circ} \mathrm{C}$ (décembre 1987), la température minimale moyenne annuelle étant de $21,66^{\circ} \mathrm{C}$ avec un écart-type de $1,04^{\circ} \mathrm{C}$. 


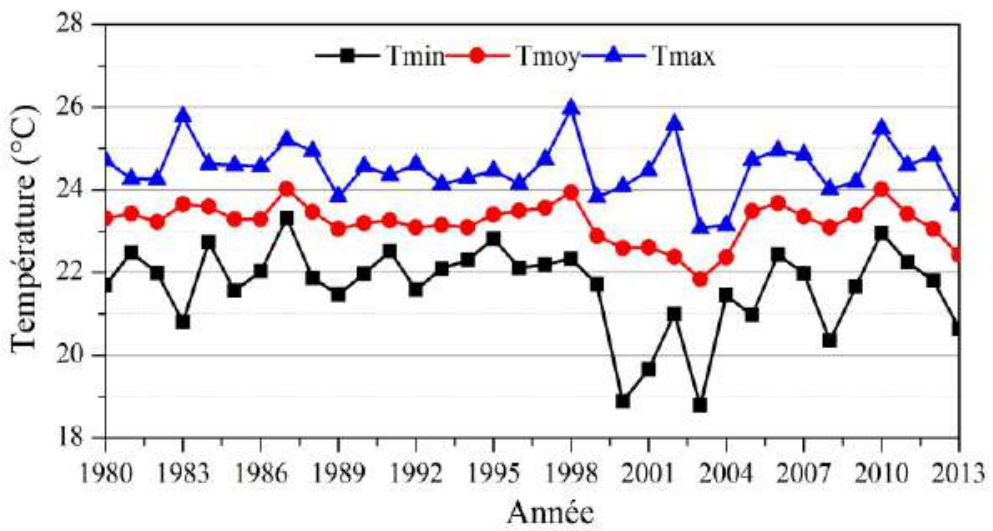

Figure 6: Evolution des températures moyennes annuelles (Tmoy), maximales (Tmax) et minimales (Tmin) de la station d'Adiopodoumé entre 1980 et 2013

\subsubsection{Variabilité des débits de la rivière Banco}

Les résultats de l'application des tests de rupture (tableau 1) montrent une rupture de stationnarité en 1996 dans la série hydrométrique avec un niveau de significativité $\alpha=0,01$. Le débit moyen interannuelle avant l'année de rupture (1996) est inférieure à celle de la période post-rupture. En effet, avant la rupture, le débit moyen était de $0,93 \mathrm{~m}^{3} / \mathrm{s}$ et après la rupture il est passé à $1,414 \mathrm{~m}^{3} / \mathrm{s}$. Cette date de rupture est confirmée par la distribution interannuelle des indices centré-réduits de pluie (figure 7).

L'évolution des indices hydrométriques montre une importante variation de l'écoulement de la rivière Banco. Cette grande variabilité se traduit par un écart de $1,879 \mathrm{~m}^{3} / \mathrm{s}$ entre la plus forte valeur du module annuel $\left(2,428 \mathrm{~m}^{3} / \mathrm{s}\right.$ en 2013$)$ et la plus faible $\left(0,549 \mathrm{~m}^{3} / \mathrm{s}\right.$ en 1987). Ainsi, la figure 7 fait ressortir de façon nette une période sèche de 1980 à 1996 et une période humide de 1997 à 2013.

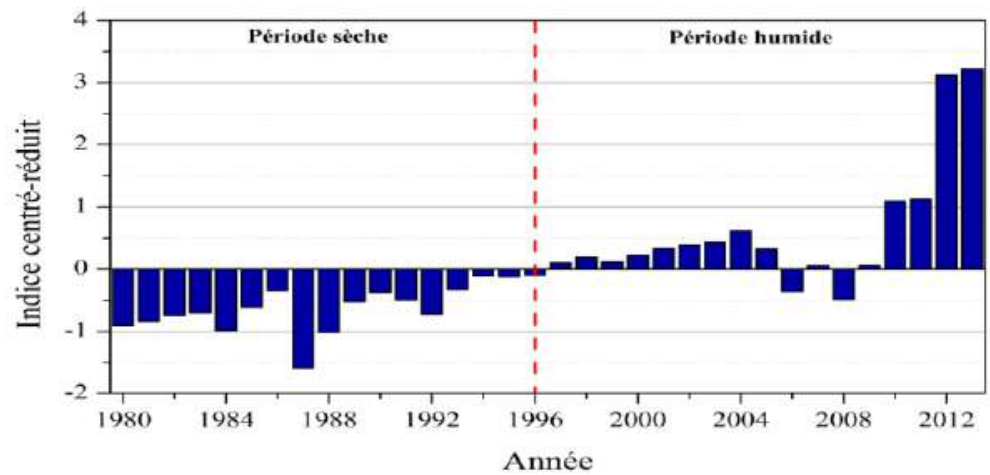

Figure 7: Evolution des indices hydrométriques du Banco à l'école forestière (1980 à 2013)

La figure 8 met en évidence les perturbations produites dans les écoulements pendant la période d'étude. Elle se traduit en général par une 
augmentation des débits dans le temps et se divise en deux parties bien distinctes. En effet, de 1980 à 1996, l'écoulement est faible, avec un débit moyen de $0,93 \mathrm{~m}^{3} / \mathrm{s}$. Sur la seconde période $1997-2013$, il croît et atteint 1,414 $\mathrm{m}^{3} / \mathrm{s}$. Le débit annuel moyen est de $1,172 \mathrm{~m}^{3} / \mathrm{s}$ à la station d'étude sur la période 1980-2013. Les années à forts déficits d'écoulement comme 1984, 1987 et 1988 sont observées sur la période 1980-1996 où les débits sont en baisse. En revanche, la période située entre 1997 et 2013 est marquée par une forte hausse du débit.

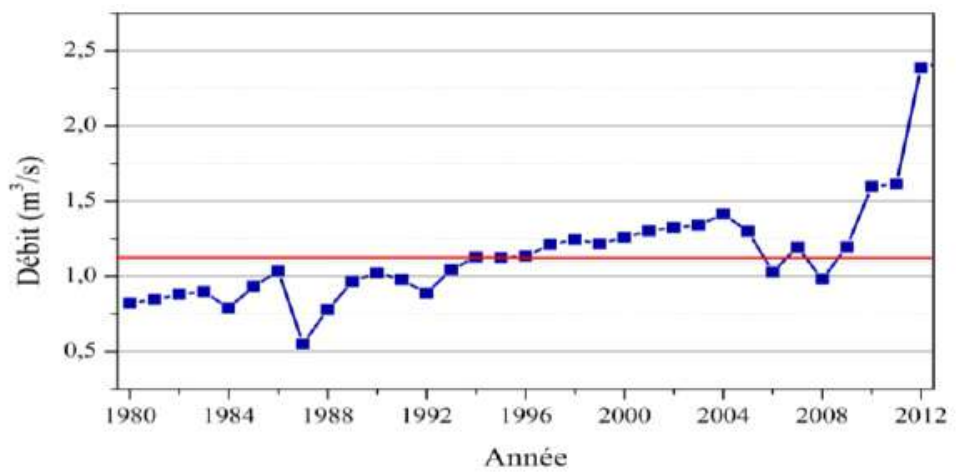

Figure 8: Evolution du débit moyen interannuel du Banco à l'école forestière (1980 à 2013)

L'hydrogramme mensuel du Banco de la chronique étudiée montre une rivière présentant des périodes de hautes eaux et de basses eaux bien distinctes. L'hydrogramme est bimodal. Les basses eaux concernent les mois de janvier à avril et d'août à septembre. Tandis que les hautes eaux apparaissent de mai à juillet et de octobre à novembre. Les débits de pointe ont lieu en juin, octobre et novembre, périodes qui correspondent aux épisodes pluvieux de la grande et de la petite saison des pluies. Les eaux commencent à monter à partir du mois de mai pour atteindre leur maximum au mois de juin. Au cours des premiers mois de hautes eaux (de mai à juillet), les débits augmentent de façon graduelle, jusqu'au maximum qui survient au mois de juin. Le débit minimum est atteint pendant le mois de janvier. De décembre à avril, l'étiage s'installe. Cette période est donc synchrone de la grande saison sèche (figure 9).

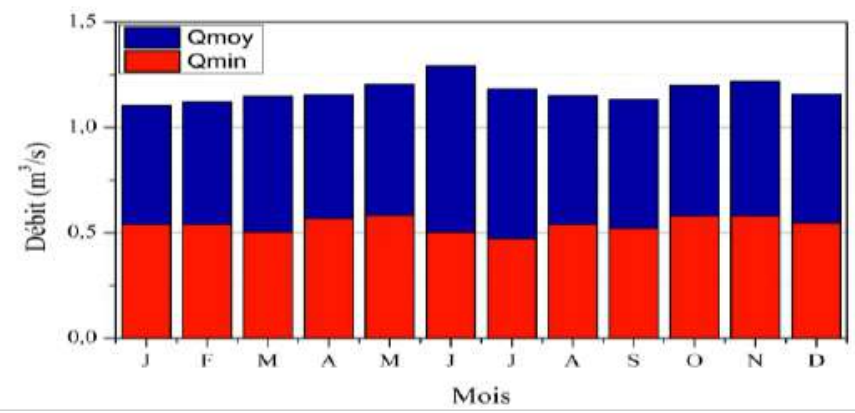

Figure 9:Variation saisonnière des débits moyens mensuels et des débits minimums mensuels du Banco à l'école forestière (1980 à 2013) 
Les coefficients mensuels de débits (C.M.D) servent à déterminer les variations inter-saisonnières. Ainsi, les mois de décembre et d'avril préalablement en hautes eaux passent en basses eaux après la rupture (1996). Tandis que le mois d'août passe de la période des basses eaux à celle des hautes eaux (Figure 10).

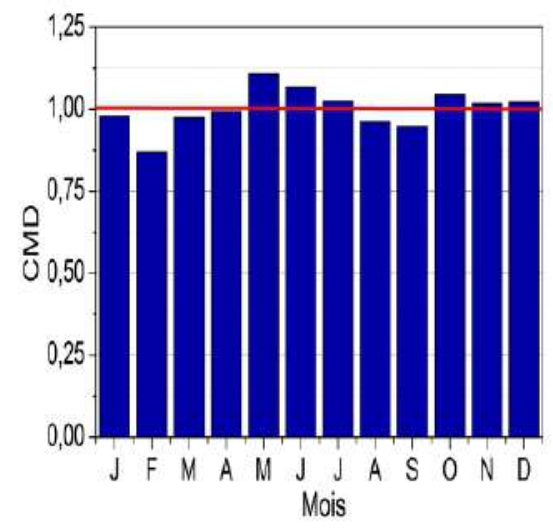

a)

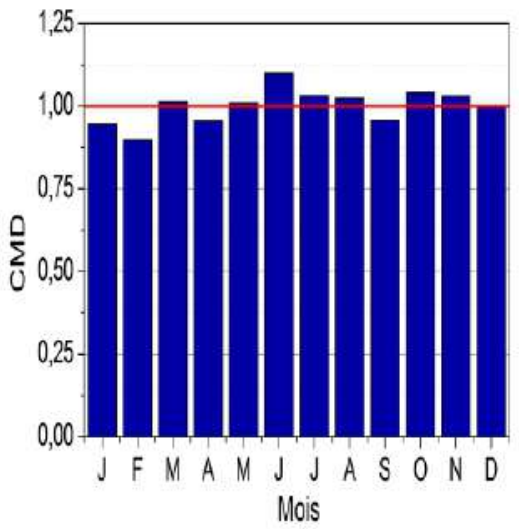

b)

Figure 10: Coefficients mensuels de débits (CMD) avant la rupture(a) et après la rupture(b)

\subsubsection{Estimation des tendances dans les séries hydroclimatiques du bassin versant de la rivière Banco}

\subsubsection{Tendance des précipitations}

Le tableau 2 montre que la tendance globale du point de vue interannuel est stationnaire. Du point de vue mensuel, de fortes variations dans la répartition des précipitations ont été enregistrées entre 1980 et 2013. La tendance est à la baisse en avril, mai, juin, août et octobre avec des pentes respectives de $-0,696 ;-0,570 ;-0,163 ;-0,947$. Tandis que, des tendances à la hausse ont été observées au mois de novembre avec une pente de 1,844 et de décembre avec une pente de 1,472. En ce qui concerne les pluies saisonnières, seule la grande saison des pluies présente une tendance à la baisse avec une pente de $-0,056$.

Tableau 2:Tendances dans les séries de pluie au moyen du test de Mann-Kendall et de l'estimateur de pente Sen.

\begin{tabular}{|c|c|c|c|c|c|c|c|}
\hline Echelle temporelle & Min & Max & Moyenne & $\begin{array}{c}\text { Ecart } \\
\text { type }\end{array}$ & $\begin{array}{c}\text { CV } \\
(\%)\end{array}$ & $\begin{array}{c}\text { MK } \\
\text { test }\end{array}$ & $\begin{array}{c}\text { Pente de } \\
\text { Sen }\end{array}$ \\
\hline Janvier & 0 & 81,4 & 24,67 & 26,28 & 106,5 & 0,445 & 0,0375 \\
\hline Février & 0,7 & 102,6 & 40,56 & 27,31 & 67,32 & 0,059 & 0,0588 \\
\hline Mars & 5,5 & 251,9 & 91,72 & 50,79 & 55,37 & 0,237 & 0,1524 \\
\hline Avril & 16,6 & 253,9 & 134,21 & 67,79 & 50,51 & $-0,504$ & $-0,6958$ \\
\hline Mai & 94,7 & 475,2 & 231,71 & 86,60 & 37,37 & $-0,385$ & $-0,5697$ \\
\hline juin & 141,6 & 905 & 387,52 & 143,14 & 36,94 & $-0,059$ & $-0,125$ \\
\hline
\end{tabular}




\begin{tabular}{|c|c|c|c|c|c|c|c|}
\hline Juillet & 0 & 440 & 136,01 & 129,52 & 95,23 & 0,133 & 0,2105 \\
\hline Août & 2,9 & 261,8 & 46,70 & 58,09 & 124,4 & $-0,474$ & $-0,1633$ \\
\hline Septembre & 3,4 & 592,5 & 92,12 & 123,98 & 134,6 & 0,534 & 0,5077 \\
\hline Octobre & 5,5 & 389,5 & 180,23 & 99,59 & 55,26 & $-0,445$ & $-0,9471$ \\
\hline Novembre & 30,5 & 247,5 & 130,29 & 59,10 & 45,36 & 1,512 & 1,8444 \\
\hline Décembre & 0 & 233,2 & 74,19 & 52,41 & 70,64 & $1,66 *$ & 1,4722 \\
\hline $\begin{array}{c}\text { Grande saison } \\
\text { sèche (DJFM) }\end{array}$ & 0 & 251,9 & 231,13 & 80,13 & 34,67 & 1,393 & 0,3697 \\
\hline $\begin{array}{c}\text { Grande saison des } \\
\text { pluies (AMJJ) }\end{array}$ & 0 & 905 & 889,45 & 282,996 & 31,82 & $-0,059$ & $-0,0562$ \\
\hline $\begin{array}{c}\text { Petite saison sèche } \\
\text { (AS) }\end{array}$ & 2,9 & 592,5 & 138,83 & 154,05 & 110,96 & 0,119 & 0,0286 \\
\hline $\begin{array}{c}\text { Petite saison des } \\
\text { pluies (ON) }\end{array}$ & 5,5 & 389,5 & 310,52 & 113,29 & 34,48 & 0,296 & 0,4375 \\
\hline Annuelle & 3109,8 & 4277,4 & 3566,44 & 290,14 & 8,17 & 0,208 & 0,0908 \\
\hline
\end{tabular}

*tendance significative au seuil de 0,1.

\subsubsection{Tendances des températures}

Le tableau 3 présente les résultats du test de Mann-Kendall des températures mensuelles, annuelles et saisonnières (minimum, maximum et moyenne) et leur tendance sur la période 1980-2013. La tendance linéaire de la température est à la baisse avec une pente de -0,01 (Tmoy), -0,019 (Tmin) et -0,009 (Tmax). L'importance de l'évolution de la température à partir de la pente de Sen confirme que la température moyenne dans le bassin versant a globalement baissé de $1,4^{\circ} \mathrm{C}$ au cours des 34 dernières années. Les températures minimales ont diminué de $3^{\circ} \mathrm{C}$ et les températures maximales de $1,2^{\circ} \mathrm{C}$. Ainsi, les températures baissent au fil du temps. Le test de MannKendall a montré également une tendance à la baisse statistiquement significative au niveau $\alpha=0,05$ au niveau des températures minimales de la grande saison des pluies.

Tableau 3:Statistique des températures mensuelles, annuelles et saisonnières (minimum, maximum et moyenne) et leur tendance par le test de Kendall et l'estimateur de pente Sen

\begin{tabular}{|c|c|c|c|c|c|}
\hline Echelle temporelle & Moyenne & $\begin{array}{c}\text { Ecart } \\
\text { type }\end{array}$ & $\mathbf{C V}(\%)$ & MK test & $\begin{array}{c}\text { Pente de Sen } \\
\text { (E-03) }\end{array}$ \\
\hline $\begin{array}{c}\text { Grande saison } \\
\text { sèche(Tmax) }\end{array}$ & 24,40 & 0,63 & 2,57 & $-0,07$ & $-0,57$ \\
\hline $\begin{array}{c}\text { Grande saison } \\
\text { sèche(Tmoy) }\end{array}$ & 23,41 & 0,54 & 2,29 & $-1,07$ & $-9,16$ \\
\hline $\begin{array}{c}\text { Grande saison } \\
\text { sèche(Tmin) }\end{array}$ & 22,10 & 1,09 & 4,90 & $-0,59$ & $-13,44$ \\
\hline $\begin{array}{c}\text { Grande saison des } \\
\text { pluies(Tmax) }\end{array}$ & 24,20 & 0,62 & 2,55 & $-1,542$ & $-16,89$ \\
\hline $\begin{array}{c}\text { Grande saison des } \\
\text { pluies(Tmoy) }\end{array}$ & 23,35 & 0,62 & 2,67 & $-1,98$ & $-14,33$ \\
\hline $\begin{array}{c}\text { Grande saison des } \\
\text { pluies(Tmin) }\end{array}$ & 22,50 & 0,86 & 3,85 & $-1,98 * *$ & $-20,03$ \\
\hline $\begin{array}{c}\text { Petite saison } \\
\text { sèche(Tmax) }\end{array}$ & 22,90 & 0,46 & 2,01 & $-0,13$ & $-1,33$ \\
\hline
\end{tabular}




\begin{tabular}{|c|c|c|c|c|c|}
\hline $\begin{array}{c}\text { Petite saison } \\
\text { sèche(Tmoy) }\end{array}$ & 22,54 & 0,52 & 2,29 & $-0,74$ & $-6,74$ \\
\hline $\begin{array}{c}\text { Petite saison } \\
\text { sèche(Tmin) }\end{array}$ & 22,22 & 0,64 & 2,87 & $-1,47$ & $-1,60$ \\
\hline $\begin{array}{c}\text { Petite saison des pluies } \\
\text { (Tmax) }\end{array}$ & 23,4 & 0,489 & 2,09 & $-0,119$ & $-2,36$ \\
\hline $\begin{array}{c}\text { Petite saison des pluies } \\
\text { (Tmoy) }\end{array}$ & 23,21 & 0,494 & 2,13 & $-0,178$ & $-2,23$ \\
\hline $\begin{array}{c}\text { Petite saison des pluies } \\
\text { (Tmin) }\end{array}$ & 23 & 0,533 & 2,32 & $-0,549$ & $-6,51$ \\
\hline Tmax (annuelle) & 24,52 & 0,644 & 2,63 & $-0,682$ & $-9,14$ \\
\hline Tmoy (annuelle) & 23,21 & 0,485 & 2,09 & $-1,423$ & $-1,01$ \\
\hline Tmin (annuelle) & 21,66 & 1,043 & 4,82 & $-1,127$ & $-1,87$ \\
\hline
\end{tabular}

**tendance significative au seuil de 0,05.

\subsubsection{Tendances des débits}

Le test de Mann-Kendall a décelé un changement dans l'écoulement entre les périodes 1980-1996 et 1997-2013. Cependant, les tendances dans les débits moyens annuels, mensuels et saisonniers sont toutes significatives à 0,01 (Tableau 4). Après avoir détecté la présence d'une tendance significative, sa magnitude est estimée à travers la pente de Sen et le taux de changement. Sur la période 1980-2013, la valeur de 0,748 est enregistrée (coefficient de Sen) et un taux de changement positif de $71,25 \%$. Cette pente montre l'ampleur de la tendance, alors que le taux de changement observé montre une forte hausse des écoulements, au fil du temps, se traduisant par des taux positifs d'amplitude de changement.

Tableau 4:Statistique des débits mensuels, annuels et saisonniers (minimums, maximums et moyennes) et leur tendance par le test de Kendall et l'estimateur de pente Sen

\begin{tabular}{|c|c|c|c|c|c|c|c|}
\hline $\begin{array}{c}\text { Echelle } \\
\text { temporelle }\end{array}$ & Min & Max & Moyenne & $\begin{array}{c}\text { Ecart } \\
\text { type }\end{array}$ & $\begin{array}{c}\text { CV } \\
(\%)\end{array}$ & MK test & $\begin{array}{c}\text { Pente de Sen } \\
\text { (E-02) }\end{array}$ \\
\hline Janvier & 0,54 & 2,27 & 1,10 & 0,36 & 30,72 & 4,71 & 2,43 \\
\hline Février & 0,54 & 2,33 & 1,12 & 0,38 & 32,42 & 4,83 & 2,77 \\
\hline Mars & 0,50 & 2,46 & 1,15 & 0,40 & 34,56 & 4,86 & 2,91 \\
\hline Avril & 0,57 & 2,29 & 1,15 & 0,40 & 33,87 & 4,42 & 2,47 \\
\hline Mai & 0,58 & 2,75 & 1,21 & 0,43 & 36,35 & 3,76 & 2,03 \\
\hline juin & 0,50 & 2,83 & 1,29 & 0,46 & 38,91 & 5,66 & 2,64 \\
\hline Juillet & 0,47 & 2,48 & 1,18 & 0,42 & 36,01 & 5,04 & 2,31 \\
\hline Août & 0,54 & 2,54 & 1,15 & 0,44 & 37,29 & 5,10 & 2,71 \\
\hline septembre & 0,52 & 2,50 & 1,13 & 0,38 & 32,08 & 5,28 & 2,49 \\
\hline octobre & 0,580 & 2,97 & 1,20 & 0,45 & 38,31 & 5,28 & 2,71 \\
\hline
\end{tabular}




\begin{tabular}{|c|c|c|c|c|c|c|c|}
\hline Novembre & 0,58 & 3,01 & 1,22 & 0,49 & 41,72 & 5,23 & 2,45 \\
\hline Décembre & 0,55 & 2,37 & 1,16 & 0,37 & 31,83 & 5,19 & 2,37 \\
\hline $\begin{array}{c}\text { Basses } \\
\text { Eaux 1 }\end{array}$ & 0,56 & 2,39 & 1,13 & 0,37 & 32,00 & 4,95 & 2,67 \\
\hline $\begin{array}{c}\text { Hautes } \\
\text { Eaux 1 }\end{array}$ & 0,52 & 2,69 & 1,23 & 0,42 & 35,49 & 5,87 & 2,17 \\
\hline $\begin{array}{c}\text { Basses } \\
\text { Eaux 2 }\end{array}$ & 0,53 & 2,43 & 1,14 & 0,40 & 33,96 & 5,19 & 2,68 \\
\hline $\begin{array}{c}\text { Hautes } \\
\text { Eaux 2 }\end{array}$ & 0,57 & 2,69 & 1,19 & 0,42 & 36,18 & 5,43 & 2,54 \\
\hline Annuelle & 0,55 & 2,43 & 14,06 & 0,39 & 33,28 & 5,84 & 7,48 \\
\hline
\end{tabular}

\section{Discussion}

Cette étude révèle une certaine stationnarité de la pluviométrie à la station d'Adiopodoumé entre 1980 et 2013. Ce comportement de la pluie dans la zone d'étude s'inscrit dans le cadre du littoral ivoirien qui se distingue de celui de régions intérieures. En effet, selon Paturel et al.(1997) les anomalies pluviométriques de la zone littorale sont moins intenses. Cependant, la détermination des indices pluviométriques et l'année de rupture a permis de constater, une continuité de la récession pluviométrique établie depuis 1970. Selon Brou (1997), dès les années 1970, la baisse de la pluviométrie prend une importance particulière dans la zone forestière, où la quasi-totalité des stations présente un déficit. La diminution de la pluviométrie s'accentue encore, au cours de la décennie 1980 (Brou, 2005). En revanche, l'indice Standardisé des Précipitations (SPI) entrevoit une reprise pluviométrique à partir de l'année 2000. Cet indice a révélé plus d'années humides que sèches. Cela confirme que la pluie n'est pas normalement distribuée temporellement. La variation interannuelle de la pluviométrie est caractérisée ainsi par une période sèche (1980-1999) et une période humide (2000-2013). La faible pluviométrie de la période 1980-1999 est contenue dans les décennies de sècheresse (1970-1979, 1980-1989, et 1990-1999) déterminées par Goula et al. (2006). Ainsi, les changements de la mousson, la température de surface de l'océan Atlantique et la déforestation sont signalés par Soro et al. (2016) comme responsables de cette diminution des précipitations. Selon ces auteurs, la réduction de la mousson provoque une diminution des précipitations. Comme d'autres études l'ont montré (Leroux, 1983; Suchel, 1988; Fontaine et al., 1992), les variations saisonnières de la pluviométrie de la zone intertropicale s'expliquent surtout par la dynamique spatio-temporelle de la ZCIT. En effet, l'extension septentrionale de la mousson provoque une augmentation de la pluviométrie. En revanche, une position australe de la ZCIT occasionne un déficit pluviométrique. Cette baisse de la pluviométrie s'explique aussi par la variation des températures de surface océanique (Charney et Shukla, 1981). 
Monteny (1986) aborde dans le même sens en affirmant que la masse d'air océanique atlantique contribue en moyenne au tiers des précipitations du pays, le reste proviendrait de l'évaporation du système forestier. On pourrait donc affirmer que la baisse des précipitations serait liée à un réchauffement anormal de la surface de l'océan atlantique. En effet, cela est dû aux interactions entre le flux de mousson et le flux de vapeur d'eau d'origine océanique (Viltard et al., 1998; Diedhiou et al.,2001). Kouadio et al. (2002) montrent qu'un refroidissement des températures de surface de l'océan atlantique au début de la grande saison des pluies (Mai), précède une hausse des précipitations sur le littoral ivoirien.

Par ailleurs, la déforestation a un impact significatif sur la circulation atmosphérique qui caractérise le flux de mousson. Ainsi, la surexploitation des zones forestières bordant l'océan atlantique contribue à la baisse de la pluviométrie. Chatelain et al. (1996) affirment que la modification des précipitations dès 1960 correspond au début de la déforestation massive en Côte d'Ivoire vers 1958. Ce phénomène est très visible sur le bassin versant du Banco, là où la forêt humide a été en partie remplacée par des habitats, des plantations et des jachères (Sako et al., 2013; Tia et al., 2015). Cette régression de la superficie forestière semble donc avoir contribué à la diminution de la pluviométrie sur le bassin. A partir de l'année 2000, la station d'Adiopodoumé est marquée par une reprise pluviométrique. Cette hausse a été mentionnée par Brou et al. (2007) dans le Sud forestier ivoirien.

La température moyenne interannuelle sur la période d'étude a varié de 21 à $24^{\circ} \mathrm{C}$. Ces valeurs sont similaires à celles déjà observées par Kouassi et al. (2005) à Abidjan. D’une manière générale, la température est demeurée supérieure à la normale sauf pour la période 1999-2004. Selon plusieurs études, la Côte d'Ivoire s'est réchauffée en moyenne de $0,3^{\circ} \mathrm{C}$ depuis 1990 (Brou et al, 2007; Ochou et al., 2007). En effet, l'augmentation de l'énergie solaire au sol d'une part et l'action des gaz à effet de serre d'autre part favorisent la hausse de la température. En saison sèche la température de l'air ambiante est élevée; ce qui entraine le réchauffement des couches d'eaux superficielles (Chantraine, 1980) et la hausse de l'évaporation (Girard, 1961). L'apparition de la grande saison de pluie entraîne un adoucissement du climat, avec une baisse sensible de la température dans les mois de juin, juillet, août et septembre. Par ailleurs, en raison de la couverture nuageuse, de l'intensification des pluies et par suite de la fraicheur, de l'humidité; les températures minimales sont enregistrées en saison pluvieuse. Un changement de tendance marqué est observé entre 1999 et 2004. Cet intervalle de temps est caractérisé par une baisse de la température alors que la pluviométrie augmente. Il est donc probable que les variations de précipitations et de températures soient liées, directement ou indirectement. Entre 1980 et 1998, les températures étaient stables. En 1999, il y a une chute puis, à partir de 2004, 
une tendance au réchauffement est constatée. Ainsi, le changement de tendance au réchauffement planétaire observé à la fin des années 70 (IPCC, 2007) a un effet retard dans le bassin versant du Banco. En Côte d'Ivoire, la température a un taux moyen d'augmentation de $1,6^{\circ} \mathrm{C}$ de 1960 à 2010 (PNUD, 2013). Cette augmentation de la température serait le résultat de l'accroissement des concentrations des gaz à effet de serre. La situation particulière d'Abidjan pourrait s'expliquer par la très forte densité d'industrie dans la ville et à sa périphérie.

L'analyse hydrométrique permet de constater avant 1996 une baisse importante des écoulements au niveau de la station du Banco. Depuis 1970, les modules annuels de plusieurs cours d'eau sont inférieurs à la moyenne générale calculée sur la période 1950-2000 (Savané et al., 2001). Les résultats des tests statistiques de détection des tendances dans la série de modules annuels concordent avec ceux observés sur la pluviométrie annuelle. Pour certains auteurs (Mahé et Olivry, 1995; Briquet et al., 1997; Mahé et al., 2001), l'exploitation de cet écart entre déficits pluviométriques viendrait d'une contribution réduite des apports d'origine souterraine à l'écoulement de surface depuis les années 1970. En effet, les réserves d'eau souterraine diminuaient au fur et à mesure que le déficit pluviométrique se prolongeait. Selon Goula et al. (2006), la baisse de la pluviométrie provoque la diminution du niveau piézométrique qui entraine celle des débits de base. Par ailleurs, la succession d'années à pluviométrie déficitaire marque aussi négativement l'écoulement. Il y a reprise des écoulements à partir de l'année hydrologique 1997-1998. Cette bonne relation entre module annuel des débits et précipitations pourrait être liée à la taille réduite $\mathrm{du}$ bassin $\left(53 \mathrm{~km}^{2}\right)$, à l'importance des pluies dans la zone littorale, mais aussi à la faible reprise évaporatoire du fait de l'adoucissement de la température.

\section{Conclusion}

Dans cette étude, il s'agissait de montrer l'existence d'une variabilité climatique, à partir des fluctuations et des tendances dans les données hydroclimatiques (pluies, températures et débits). Les résultats obtenus à partir de l'indice Standardisé des Précipitations (SPI) et des tests de rupture (Cusum et t-student) sur la variabilité pluviométrique du bassin versant de la rivière Banco sont marqués par une période sèche et une période humide respectivement de 1980 à 1999 et de 2000 à 2013. Par contre, avec le test de Mann-Kendall, les précipitations sont stationnaires sur toute la chronique (1980-2013). La variation interannuelle de température sur la période 19802013 a révélé deux périodes chaudes (1980-1998 et 2005-2013) séparées par une période froide (1999 et 2004). La pente de Sen a enregistré une baisse de la température moyenne de $1,42^{\circ} \mathrm{C}$ au cours des 34 dernières années dans le bassin versant. Du point de vue hydrométrique, la variation de l'écoulement 
de la rivière Banco présente un écart important de $1,879 \mathrm{~m}^{3} / \mathrm{s}$. Le régime hydrologique se manifeste de 1980 à 1996 par une période déficitaire, avec un débit moyen de $0,93 \mathrm{~m}^{3} / \mathrm{s}$, et de 1997-2013 par une période excédentaire dont le débit moyen est de $1,414 \mathrm{~m}^{3} / \mathrm{s}$. Les tendances dans les débits moyens calculés sont positives et significatives au seuil de 0,01 avec un taux de changement annuel positif $(71,25 \%)$. Ce taux exprime une forte hausse des écoulements, au fil du temps. Les résultats de cette étude peuvent servir à amorcer des études futures visant à montrer l'existence des relations directes entre les tendances pluviométriques et la dynamique de l'occupation du sol.

\section{References :}

1. Bricquet J. P., Bamba F., Mahé G., Touré M. et Olivry J. C. (1997). Evolution récente des ressources en eau de l'Afrique atlantique. Journal of Water Science, Vol.10, $\mathrm{N}^{\circ}$ 3, pp. 321-337.

2. Briquet J. P., Mahe G., Bamba F., Olivry J. C. (1996). Changements climatiques récents et modification du régime hydrologique du Niger à Koulikoro (Mali).Colloque J. Rodier, pp 157-166.

3. Brou Y. T. (1997). Analyse et dynamique de la pluviométrie dans le sud forestier ivoirien: recherche de corrélations entre les variables climatiques et les variables liées aux activités anthropiques. Thèse de Doctorat, Université de Cocody, Abidjan (Côte d'Ivoire), $211 \mathrm{p}$.

4. Brou Y. T. (2005). Climat, mutations socio-économiques et paysages en Côte d'Ivoire. Mémoire de synthèse des activités scientifiques présentées en vue de l'obtention de l'Habilitation à Diriger des Recherches, Université des Sciences et Techniques de Lille(France), 212 p.

5. Brou Y. T. et Chaléard J. L. (2007). Visions paysannes et changements environnementaux en Côte d'Ivoire. Annales de géographie, Vol.653, pp 65-87.

6. Chantraine J.-M. (1980). La lagune Aby (Côte d'Ivoire) : morphologie, hydrologie, paramètres physico-chimiques. Abidjan : CRO, Vol.11, $\mathrm{N}^{\circ}$, pp. 39-77.

7. Charney J. G. et Shukla J. (1981). Predictability of monsoons. Monsoon Dynamics, J. Lighthill and R. P. Pearce, Eds., Cambridge University Press, pp. 99-109.

8. Chatelain C., Gautier L. et Spichiger R. (1996). Forest history fragmentation in southern Côte d'Ivoire. Biodiversity and Conservation, Vol 5, pp. 37-53.

9. Diedhiou A., Janicot S., Viltard A. et De Felice P. (2001). Composite patterns of easterly disturbances over West-Africa and the tropical Atlantic: a climatology from the 1979-1995 NCEP/NCAR reanalyses. Clim. Dyn., Vol.18, pp. 241-253. 
10. Durand J.R. et Chantraine J.M. (1982). L'environnement climatique des lagunes Ivoiriennes. Rev. Hydrobiol. Trop., Vol.15, N², pp. 85113.

11. Faye C. (2017). Variabilité et tendances observées sur les débits moyens mensuels, saisonniers et annuels dans le bassin de la Falémé (Sénégal). Hydrological Sciences Journal,Vol. 62, pp. 259-269.

12. Fontaine B. et Janicot S. (1992). Wind-field coherence and its variations over West Africa. Journal of Climate,Vol. 5, N5, pp. 512524.

13. Fossou R.M.N. , Lasm T. , Soro N. , Soro T. , Soro G., de Lasme O.Z.,Baka D., Onetie O.Z. et Orou R. (2015). Variabilité climatique et son impact sur les ressources en eaux souterraines : cas des stations de Bocanda et de Dimbokro au Centre-est de la Côte d'Ivoire (Afrique de l'ouest). Larhyss Journal, N²1, pp. 97-120.

14. Girard M. G. (1961). Etude du basin versant de la rivière Agnéby, résultats de la campagne de 1960. Service hydrologique de l'ORSTOM, 127 p.

15. Gocic M. et Trajkovic S. (2013). Analysis of changes in meteorological variables using Mann-Kendall and Sen's slope estimator statistical tests in Serbia. Global and Planetary Change, Vol. 100, pp. 172-182.

16. Goula B. T. A., Savane I., Konan B., Fadika V. et Kouadio G. B. (2006). Impact de la variabilité climatique sur les ressources hydriques des bassins de N'Zo et N'Zi en Côte d'Ivoire (Afrique tropicale humide). Vertigo, Vol.1, pp. 1-12.

17. IPCC (2007). Climate Change 2007: the physical science basis. Contribution of working group to the fourth assessment report of the intergovernmental panel on climate change. 104p.

18. Jourda J. P. (1987). Contribution à l'étude géologique et hydrogéologique de la région du Grand Abidjan (Côte d'Ivoire). Thèse de doctorat de 3ème cycle, Université scientifique, technique et médicale de Grenoble, 319 p.

19. Kendall M. G. (1955). Rank correlation methods ( $2^{\text {nd }}$ ed.). London: Griffin, 196 p.

20. Kouadio K. Y. (2002). Influence des paramètres météo-océaniques de l'Atlantique tropical sur la pluviométrie en Côte d'Ivoire. Doctorat de l'Université de Cocody-Abidjan (Côte d'Ivoire), 176 p.

21. Kouadio Y., Ochou D.A. et Servain J. (2002). Atlantic influence on the rainfall variability in Côte d'Ivoire. Geophysic Research Letters, Vol.30, pp 8005-8012.

22. Kouassi A. M., Tidou A. S. et Kamenan A. (2005). Caractéristiques hydrochimique et microbiologique des eaux de la lagune Ebrié (Côte 
d'Ivoire). Partie I: variabilité saisonnière des paramètres hydrochimiques. Agronomie Africaine, Vol.17, N², pp 117-136.

23. Kouassi K. A., Kouassi F. W., Goula B. T. A., Kouamé K. I., Dibi B. et Savané I. (2010). Conceptual model of Ivorian sedimentary costal basin: case of Abidjan Continental Terminal aquifer. European Journal of Scientific Research, Vol. 44, N³, pp 400-419.

24. Kumar V., Jain S. K. et Singh Y. (2010). Analysis of long-term rainfall trends in India. Hydrol. Sci. J., Vol. 55, pp. 484-496.

25. Leroux M. (1983). Le climat de l'Afrique tropicale. Paris: Ed. Champion, Vol. 2, 650 p.

26. Loroux B.F.E. (1978). Contribution à l'étude hydrogéologique du bassin sédimentaire côtier de Côte d'Ivoire. Thèse Université de Bordeaux I, France, 93 p.

27. Mahé G. et Olivry J. C. (1995). Variations des précipitations et des écoulements en Afrique de l'Ouest et Centrale de 1951 à 1989. Sécheresse, Vol.6, N¹, pp. 109-117.

28. Mahé G., L'Hote Y., Olivry J. C. et Wotling W. (2001). Trends and discontinuities in regional rainfall of West and Central Africa. Hydrological Sciences Journal, Vol.46, N², pp. 211- 226.

29. Mann H.B. (1945). Non-parametric tests against trend. Econometrica, Vol. 13, pp 163-171.

30. Monteny B. et Zeller B. (1986). Interactions végétation-atmosphère et leurs impacts sur les caractéristiques climatiques. In :Toure Y.M. (ed.)Climat et développement. Paris : ORSTOM, pp 157-166

31. Nicholson S. E. (1980). The nature of rainfall fluctuations in subtropical West Africa. Monthly Weather Review, Vol.108, N4, pp. 473-487.

32. Ochou A. D., Nzeukou A. et Sauvageot H. (2007). Parametrization of drop size distribution with rain rate. Vol. 84, pp. 58-66.

33. Patle G. T. et Libang A. (2014). Trend analysis of annual and seasonal rainfall to climate variability in North-East region of India. journal of Applied and Natural Science, Vol. 6, $\mathrm{N}^{\circ}$ 2, pp. 480-483.

34. Paturel J. E., Serval E., Lubes-Niel H. et Delallre M. O. (1997). Variabilité climatique et analyse de séries pluviométriques de longue durée en Afrique de l'Ouest et centrale non sahélienne. C.R. Acad. Sci., Paris 325, série Ila, pp. 779-782.

35. PNUD (2013). Rapport annuel du programme pays. Bulletin périodique, $18 \mathrm{p}$.

36. Sako N., Beltrando G., Atta K. L., N'da D. H. et Brou T. (2013). Dynamique forestière et pression urbaine dans le Parc national du Banco (Abidjan, Côte d'Ivoire),VertigO - la revue électronique en 
sciences de l'environnement, Vol. 13, $\mathrm{N}^{\circ} 2$ : http://vertigo.revues.org/14127

37. Savane I., Coulibaly K. M. et Gioan P. (2001). Variabilité climatique et ressources en eaux souterraines dans la région semi-montagneuse de Man. Sécheresse, Vol.12, N4,pp. 231-237.

38. Sen P. K. (1968). Estimates of the regression coefficient based on Kendell's tau.J.Am.Stat.Assoc., Vol. 63, pp. 1379-1389.

39. Servat E., Paturel J.E., Lubès H., Kouamé B., Ouedraogo M. et Masson J.M. (1997). Climatic variability in humid Africa along the Gulf of Guinea. Part one: Detailed analysis of the phenomenon in Côte d'Ivoire. Journal of Hydrology, Vol.191, pp.1-15.

40. Soro G. E., Noufé D., Goula B. T. A et Shorohou B. (2016). Trend Analysis for Extreme Rainfall at Sub-Daily and Daily Timescales in Côte d'Ivoire. Climate, $15 \mathrm{p}$.

41. Suchel J.B. (1988). Les climats du Cameroun. These de Doct. d'état, University of St Etienne, Saint-Etienne, Vol. 4, 1188 p.

42. Tia L. et Dago D. R. (2015). Morcellement d'une aire protégée en agglomération urbaine : le cas du parc national du Banco (Côte d'Ivoire). Cahiers de géographie du Québec, Vol.59, №168, pp. 349376. https://doi.org/10.7202/1037254ar

43. Viltard A., Oubuih J., de Felice P.et Laurent H. (1998). Rainfall and 6-9 day wave-like disturbance in West Africa during summer 1989.Meteor. Atmos. Phys., Vol. 66, pp.229-234.

44. WCMC (1991). Guide de la Diversité Biologique de la Côte d'Ivoire. WCMC, Cambridge, $25 \mathrm{p}$

45. Yao F.Z., Reynard E., Ouattara I., N'go Y.A., Fallot J-M. et Savané I. (2018). A New Statistical Approach to Assess Climate Variability in the White Bandama Watershed, Northern Côte d'Ivoire. Atmospheric and Climate Sciences, Vol. 8, pp 410-430. https://doi.org/10.4236/acs.2018.84027

46. Zerouali B., Mesbah M., Chettih M., Abda Z. et Djemai M. (2015). Analyse et cartographie des tendances pluviométriques dans le bassin versant de l'Oued Sébaou (Nord-Centre de l'Algérie).International Conference on African Large River Basins Hydrology Hammamet, Tunisia. 